Curr Dir Psychol Sci. 2013 October 1; 22(5): 400-406. doi:10.1177/0963721413488889.
The Developmental Effects of Early Life Stress: An Overview of
Current Theoretical Frameworks
Camelia E. Hostinar, Ph.D. and Megan R. Gunnar, Ph.D.
Institute of Child Development, University of Minnesota
Abstract
The field of psychobiology has two major theories for talking about stress and health: the allostatic
load model, which grew out of biological and neuroscience approaches to understanding health
and disease, and the adaptive calibration model, which developed out of an explicitly
evolutionary-developmental framework. Both are based on assumptions that the brain coordinates
a distributed and dynamic set of neural circuits that regulate behavior and stress physiology to help
the organism adapt to the demands of the environment. Both models support the notion that
experiences early in life are embedded into the regulation of stress systems in ways that shape the
organism's future responses. These two paradigms differ in their emphasis on whether changes in
how stress systems function are viewed as adaptive or maladaptive. The goal of this review is to
identify the strengths and weaknesses of each framework and to discuss some implications for
future studies and for policy.

\title{
Keywords
}

stress; early adversity; adaptation

Childhood adversity is emerging as a significant risk factor for mental and physical illness later in life (Shonkoff, Boyce, \& McEwen, 2009). The field of psychobiology has two types of theoretical orientations to explain how early life stress "gets under the skin" to set individuals on pathways toward physical and mental health problems. The role of the brain in coordinating behavioral and physiological stress systems to adapt to ongoing demands of the external and internal environment is accounted for in both types. Both allow for an understanding of these processes as resulting in individual differences in stress reactivity that shape the organism's responses to later conditions. These two orientations are different in their frame of reference for adaptation and, as a result, their emphasis on whether patterns of stress responses to chronic adversity are adaptive versus maladaptive. Our goals are to examine the strengths and limitations of each approach and to identify future directions that, we hope, will inform science and policy.

Corresponding Author: Camelia E. Hostinar, 51 East River Road, Minneapolis, MN 55455; hosti002@umn.edu.

Conflict of Interest Disclosure:

The authors declared that they had no conflicts of interest with respect to their authorship or the publication of this article. 


\section{The Two Models}

The allostatic load model (ALM) (McEwen, 1998, 2008; McEwen \& Stellar, 1993) was designed to explain the processes linking stress and disease by studying allostatic load, which has been defined as "the impact of wear and tear on a number of organs and tissues[, which] can predispose the organism to disease" (McEwen \& Stellar, 1993, p. 2094). Allostasis (allo $=$ variable, stasis $=$ state $)$ is defined as "achieving stability through change" (McEwen \& Wingfeld, 2003, p. 3); that is, the organism undergoes physiological changes to respond to challenges in an attempt to restore its optimal state, for instance by producing stress hormones or launching immune responses. The model suggests that even though allostasis often has short-term benefits, over long periods, these allostatic adjustments-if frequent or chronic — can take a toll on the body. That is, there are trade-offs in health and disease, and animals can adopt different behavioral strategies (e.g., low or high aggression) for coping with stress that have differential costs and benefits for the organism (Korte, Koolhaas, Wingfeld, \& McEwen, 2005). The cumulative aspect of allostasis has been termed allostatic load. Furthermore, when certain events dramatically increase the allostatic load on the organism, the term allostatic overload (McEwen \& Wingfeld, 2003) has been introduced to describe the process presumed to play a role in the development of mental and physical illness through effects on the body and the brain. The ALM describes a range of systems that mediate allostasis, including neural, endocrine, cardiovascular, autonomic, immune, and metabolic systems (see Fig. 1 and McEwen, 2008). This model has contributed significantly to the science of aging and of socioeconomic disparities in health. The continued goal of advocates of the ALM is to identify biological mechanisms that increase the risk for pathologic conditions and the role of stress systems in health and disease processes.

From the perspective of the adaptive calibration model (ACM; Del Giudice, Ellis, \& Shirtcliff, 2011; a revision of biological sensitivity to context theory—Boyce \& Ellis, 2005; Ellis \& Boyce, 2008 — and similar to differential susceptibility theory—Belsky \& Pluess, 2009), individual differences in stress reactivity are evolutionarily selected adaptations that enable the developing organism to match its phenotype to different environmental conditions (Fig. 2). This theory constitutes an evolutionary-developmental approach, attempting to enable analysis of behaviors through the lens of potential evolutionary advantages that might have facilitated their emergence and maintenance while also suggesting a role for individual developmental change across several life stages. Furthermore, the model postulates a broader role for stress-response systems in affecting a wide array of behaviors and physiological systems, including reproduction, competition, learning and growth, and so forth (see Fig. 2). This is because adversity is thought to shift life history strategies, and the theory proposes sex differences in these strategies and in how they are affected by stressors. One of the strengths of the ACM for developmentalists is that it is explicitly developmental, and it allows for the possibility that what is programmed early in life may be recalibrated later to accommodate changes in life conditions. 


\section{Where the Two Frameworks Converge}

The two theoretical views are consistent in that stress systems are understood to play a pivotal role in the organism's adaptation to the demands of the external and internal environment. By mobilizing energy and propelling the organism to avoid or cope with threat (to name just a few examples), stress responses protect the organism. Both theoretical perspectives support the idea of mediating roles of the hypothalamic-pituitary-adrenal axis and autonomic nervous system in executing these stress responses and implementing allostasis, as well as their interactions with neural, immune, and metabolic systems. Both models posit that stress responses have evolved because they increase our survival advantage. The ALM indicates that allostatic processes have probably been shaped by evolution to maximize survival in certain environments given that vital functions need to be maintained within narrow parameters for the organism to be viable (Danese \& McEwen, 2012; Korte et al., 2005). The ACM also suggests that stress systems have been evolutionarily selected for their survival advantage and further that individual differences in stress reactivity are due to an evolved ability for conditional adaptation to environmental conditions (Del Giudice et al., 2011). Both models take into account the role of stress system activity in shaping broader behavioral strategies such as aggression, parenting, and reproduction (Del Giudice et al., 2011; Korte et al., 2005).

Both models also acknowledge the biological trade-offs associated with deploying the stress-response system. For example, the ALM describes how stress-induced elevations in cortisol and inflammation accompany metabolic processes that conserve energy during stressful periods but also elevate risk for metabolic syndrome (Danese \& McEwen, 2012). Furthermore, the model suggests that stress effects on brain systems involved in threat and reward allow adaptation to adverse contexts, potentially at the cost of increasing the risk of addiction, depression, and other outcomes that may increase morbidity and mortality (Juster, McEwen, \& Lupien, 2010). The ACM borrows from life-history theory to illustrate how organisms prioritize the life domains to which they dedicate their energy (e.g., reproduction over survival or survival over growth; Ellis, Del Giudice, \& Shirtcliff, 2013). For instance, others and we have observed that children adopted from orphanages exhibit stunted growth (Gunnar, 2001; Johnson, Bruce, Tarullo, \& Gunnar, 2011). The condition is believed to be the result of interactions between the stress and growth axes, such that elevated activity of the hypothalamic-pituitary-adrenal axis decreases both the activity of the growth hormone axis and tissue sensitivity to growth hormone. These findings highlight the energetic costs of adapting and activating stress physiology.

\section{Is Adaptation to Maladaptation Adaptive? Differences Between the Models}

This meaningless title shows that the use of the term adaptive can be a source of confusion when its reference framework (public health or evolutionary biology) is not clarified. The two models are not necessarily mutually exclusive; they simply specify different benchmarks for what is considered adaptive. Much like the blind men attempting to describe the elephant in the ancient Indian parable, these models are different lenses through which to observe the phenomenon and may seem to be describing different "elephants" sometimes. The ALM is concerned with proximal mechanisms and the question of how chronic stress 
exposure affects stress-mediating systems in ways that eventually lead to disease processes. In contrast, the ACM adopts an ultimate lens, in which the critical issue is the natural evolution of physiological and behavioral systems across large spans of time, attempting to answer the question of why stress systems operate the way they do today by examining the function of variation across individuals and across development. The organism's fitness (i.e., the ability to transmit genes contributing to different patterns of adaptation to future generations) is the criterion for adaptation in the ACM. The two frameworks diverge in some ways, because allostatic overload is considered in the ALM to be detrimental to the organism (unlike allostasis, which has adaptive functions), whereas the ACM allows for the possibility of some unexamined positive outcomes associated with cumulative stress (Ellis et al., 2013).

Proponents of the ACM criticize the ALM for its overemphasis on pathology and the longterm costs of allostasis (Ellis et al., 2013); however, several decades of undeniable epidemiological findings link chronic stressors with increased risk of cardiovascular disease, metabolic syndrome, accelerated cognitive declines, and shorter lifespans (McEwen \& Gianaros, 2011). Thus, there is a societal benefit to better understanding these links to prevent disease. Supporters of the ACM might argue that these outcomes are not necessarily maladaptive from an evolutionary perspective; rather, they are simply undesirable from a societal and public health perspective. This is an ideological and not an empirical difference between the two models that is likely to remain unresolved because people and organizations who aim to promote public health drive a large portion of research. The ideological orientation one chooses to adopt will indeed shape the types of questions asked and whether these questions focus on disease or desirable outcomes that may be affected by environmental conditions, but we believe that empirical results obtained under each framework could well complement the other to show the full spectrum of outcomes associated with early life stress.

Another critique leveled against the ALM is that it specifies both hypo- and hyperarousal as dysregulated patterns, despite developmental findings that in some instances have related both low and high cortisol activity to positive outcomes. As an example, the ACM accounts for children with externalizing problems that are also more stress-reactive and consequently less likely to develop conduct problems later (Calkins \& Keane, 2009). However, the more desirable clinical outcome (low conduct problems) does not preclude undesirable health outcomes later in life as a result of frequent activation of stress mediators that can increase allostatic load. Again, it would be useful to specify the frame of reference for our benchmark for "adaptation." Furthermore, we must be cautious in extrapolating findings that link unfortunate experiences (child maltreatment, combat trauma, etc.) with hypo- or hyperarousal of stress systems, because the reverse inference is not always true (hypo- or hyperreactivity does not uniquely or necessarily point to a traumatic experience). The ACM highlights the need to better understand these nonlinear associations between physiology and behavior. We agree that psychobiological science needs to go beyond single correlations between a physiological measure and a desirable or undesirable behavioral outcome, leading to labeling of a physiological process as well regulated versus dysregulated. Proponents of the ACM would seem to agree with valid criticisms (a) that too many studies report 
"dysregulation" when a difference in cortisol levels is observed between groups, (b) that we would benefit from a value-neutral description of relations between physiology and behavior, and (c) that clear criteria are necessary to describe the stress system as "dysregulated."

Another divergence of the two models is their perspective on individual differences in the tuning of stress systems. We know that some people succumb and others thrive when confronted with similar challenges (Gunnar \& Quevedo, 2007), but researchers have yet to fully elucidate the origins of these differences. The ALM recognizes the individual differences that influence the accumulation of allostatic load and acknowledges the work on early adversity and the programming of the hypothalamic-pituitary-adrenal axis as one source of individual difference in vulnerability to allostatic overload (Danese \& McEwen, 2012), but complete mechanisms explaining these individual differences in theory have not yet been proposed.

On the other hand, the ACM is explicitly a model of evolved differences in patterns of biobehavioral responses to stressors. Its predecessor model (biological sensitivity to context theory) postulated the provocative idea that people who are highly responsive to the environment fare best under supportive conditions (better than people with low responsivity) but worst under poor conditions. These models stand in marked contrast to older diathesisstress models, which viewed certain individual characteristics as diatheses that turn into negative outcomes under stressful conditions, but do not provide advantages otherwise. Although theories such as the ACM depend on statistical interactions that are notoriously difficult to replicate, such interactions are increasingly reported (Belsky, BakermansKranenburg, \& van IJzendoorn, 2007). Furthermore, some preliminary evidence supports some of the ACM predictions on the basis of family stress in a middle childhood sample (Del Giudice, Hinnant, Ellis, \& El-Sheikh, 2012). However, the ACM does not explain or predict the biological mechanisms through which different stress reactivity phenotypes emerge.

\section{Developmental Phenomena Seeking Developmental Explanations: Future Directions}

Finally, the models diverge on the issue of development. Despite providing some useful insights, neither theory offers a complete account of the developmental psychobiology of stress systems. The ALM was not designed to explain developmental processes but might be applied to childhood stressors and, as noted, its supporters are increasingly acknowledging that the prenatal and early postnatal periods (Danese \& McEwen, 2012) and pubertal period (Romeo \& McEwen, 2006) may be sensitive periods for programming of the hypothalamicpituitary-adrenal axis and thus vulnerable periods for chronic stress exposure. However, it is likely that there is not a single sensitive period for stress systems; rather, each of the distributed systems that orchestrate behavioral and physiological responses to stressors has its own developmental history, with multiple sensitive periods involved. Proximal mechanisms need to be better characterized, and science is indeed beginning to understand some of the molecular bases of sensitive periods in development (Hensch, 2005). 
The ALM holds that disease emerges from cumulative damage on the body over time and from the programming of physiology during sensitive periods such as the first years of life (Shonkoff et al., 2009). The ACM suggests that maladaptation occurs as a result of miscalibration or mismatches between (a) early development and later development or (b) an organism's evolutionary history and the environment actually encountered (Ellis et al., 2013). There is indeed evidence that developmental miscalibration occurs (e.g., the case of prenatal programming; Barker, 1998); however, the effects of evolutionary miscalibration are more challenging to study. For instance, the ACM makes no predictions about the development of children reared in orphanages because its proponents argue that these extreme experiences are outside our evolutionary heritage (Ellis et al., 2013). If mismatches between the environments that we evolved in and current life are rare, then the failure of the ACM to account for them is nominal. However, there is a strong argument that much in modern life, particularly as it affects stress and metabolic systems, constitutes mismatches to our evolutionary heritage (Gluckman \& Hanson, 2007). Thus, failure to deal with evolutionary mismatches and assuming that common patterns are adaptive solutions that will facilitate fitness could be serious limitations of the ACM. The ALM does not address these mismatches either, but its focus on the effects of cumulative stress on individual development may orient us to understanding people who experience these extreme circumstances.

The ACM postulates a prolonged period of plasticity for the development of stress systems and many periods during which the organism is especially open to programming or calibration, including the prenatal period and first years of life, middle childhood, puberty, and even continuing into adulthood. Major transitions such as the birth of the first child or menopause are proposed to serve as potential switch points in a person's trajectory (Del Giudice et al., 2011). These are provocative claims, and it will be important to gather empirical evidence for these predictions. Furthermore, it will be important to specify exactly which periods (and which components of the stress-response system in which periods) are most plastic across development, because this may be relevant for future interventions. An example of the type of evidence needed for these hypotheses comes from Quevedo, Johnson, Loman, LaFavor, and Gunnar (2012). They showed that the morning cortisol response reflected early adversity (experienced before age 3 ) in teenagers who were in the early stages of puberty compared with those in late stages, who showed no differences in cortisol awakening responses compared with a low-stress comparison group. This may suggest a recalibration of stress systems during puberty but does not rule out prior periods of plasticity. Thus, we need a better understanding of when different aspects of the stressresponse system recalibrate, which will require a better understanding of proximal mechanisms.

An answer to this issue may emerge from recent advancements in molecular biology that have shown that environmental signals can regulate gene expression through epigenetic changes (Gilbert \& Epel, 2009), which can be both inherited by offspring and reversed under specific conditions. For instance, rodents experiencing high levels of maternal care exhibit epigenetic changes in the glucocorticoid receptor gene promoters in the hippocampus, which are important in controlling the hypothalamic-pituitary-adrenal stress response (Meaney \& Szyf, 2005). These effects are observed even if the pups are cross- 
fostered at birth (Francis, Diorio, Liu, \& Meaney, 1999), supporting the causal role of experience. Finally, there is also evidence that variations in early rearing environments produce marked differences in the epigenome in many organs and tissues of the body. Intergenerational transmission of characteristics thus happens not only through inheritance of structural genes but also through epigenetically transmitted patterns of development. Furthermore, classic evolutionary theory could not explain the emergence of variation in phenotypes caused by environmental parameters, which have been termed polyphenisms (Gilbert \& Epel, 2009). Modern evolutionary biology emphasizes developmental plasticity (West-Eberhard, 2003) and the need for a biological theory of developmental change. Such a theory would help us understand the emergence of these variations upon which natural selection ultimately operates, and recently there have been efforts to integrate these insights into the study of childhood adversity (Blair \& Raver, 2012). A deeper understanding of epigenetics and the underlying biology of developmental plasticity in stress systems will contribute to our future understanding of these individual differences.

\section{Public Policy Implications}

Both models discussed have explicit and implicit consequences when we attempt to translate the science of early life stress into public policy. Emphasizing the negative impact of stress on health shifts policy toward minimizing adverse experiences and fostering programs to help people manage stress more effectively. But without an understanding of the adaptive significance of the stress-stimulated behavior, our intervention efforts may be ineffective. For instance, callous bullying by adolescent boys who have experienced chronic stress may have persisted through evolution because of beneficial consequences on social status or access to resources (Ellis et al., 2012). Taking the behaviors away without providing an effective alternative may meet resistance and result in failed interventions or it may worsen these behaviors. Thus both perspectives may add importantly to our approach to public policy and public health.

\section{Conclusions}

The ALM and ACM are largely orthogonal. The ACM is explicitly developmental, which is a benefit for developmental researchers. However, it does not clearly delineate the proximal mechanisms through which developmental effects are instantiated. The ALM is not a developmental theory, but its proponents often point to the fetal/perinatal programming literature as one of the mechanisms through which individual differences in vulnerability to allostatic load may emerge. In truth, neither model yet provides an adequate developmental theory of stress. In this regard, both are works in progress.

\section{Acknowledgments}

This work was supported by the Eva O. Miller Fellowship to Camelia E. Hostinar and by National Institutes of Health Grants MH080905 and MH078105 to Megan R. Gunnar.

\section{References}

Barker DJP. In utero programming of chronic disease. Clinical Science. 1998; 95:115-128. [PubMed: 9680492] 
Belsky J, Bakermans-Kranenburg MJ, van IJzendoorn MH. For better and for worse: Differential susceptibility to environmental influences. Current Directions in Psychological Science. 2007; 16:300-304.

Belsky J, Pluess M. Beyond diathesis stress: Differential susceptibility to environmental influences. Psychological Bulletin. 2009; 135:885-908. [PubMed: 19883141]

Blair C, Raver CC. Individual development and evolution: Experiential canalization of self-regulation. Developmental Psychology. 2012; 48:647-657. [PubMed: 22329384]

Boyce WT, Ellis BJ. Biological sensitivity to context: I. An evolutionary-developmental theory of the origins and functions of stress reactivity. Development and Psychopathology. 2005; 17:271-301. [PubMed: 16761546]

Calkins SD, Keane SP. Developmental origins of early antisocial behavior. Development and Psychopathology. 2009; 21:1095-1109. [PubMed: 19825259]

Danese A, McEwen BS. Adverse childhood experiences, allostasis, allostatic load, and age-related disease. Physiology \& Behavior. 2012; 106:29-39.

Del Giudice M, Ellis BJ, Shirtcliff EA. The Adaptive Calibration Model of stress responsivity. Neuroscience \& Biobehavioral Reviews. 2011; 35:1562-1592. [PubMed: 21145350]

Del Giudice M, Hinnant JB, Ellis BJ, El-Sheikh M. Adaptive patterns of stress responsivity: A preliminary investigation. Developmental Psychology. 2012; 48:775-790. [PubMed: 22148947]

Ellis BJ, Boyce WT. Biological sensitivity to context. Current Directions in Psychological Science. 2008; 17:183-187.

Ellis BJ, Del Giudice M, Dishion TJ, Figueredo AJ, Gray P, Griskevicius V, Wilson DS. The evolutionary basis of risky adolescent behavior: Implications for science, policy, and practice. Developmental Psychology. 2012; 48:598-623. [PubMed: 22122473]

Ellis, BJ.; Del Giudice, M.; Shirtcliff, EA. Beyond allostatic load: The stress response system as a mechanism of conditional adaptation. In: Beauchaine, TP.; Hinshaw, SP., editors. Child and adolescent psychopathology. 2. Hoboken, NJ: Wiley; 2013. p. 251-284.

Francis D, Diorio J, Liu D, Meaney MJ. Nongenomic transmission across generations of maternal behavior and stress responses in the rat. Science. 1999; 286:1155-1158. [PubMed: 10550053]

Gilbert, SF.; Epel, D. Ecological developmental biology: Integrating epigenetics, medicine, and evolution. Sunderland, MA: Sinauer Publishers; 2009.

Gluckman, PD.; Hanson, MA. Mismatch: Why our world no longer fits our bodies. Oxford, England: Oxford University Press; 2007.

Gunnar, MR. Effects of early deprivation: Findings from orphanage-reared infants and children. In: Nelson, CA.; Luciana, M., editors. Handbook of Developmental Cognitive Neuroscience. Cambridge, MA: MIT Press; 2001. p. 617-629.

Gunnar MR, Quevedo K. The neurobiology of stress and development. Annual Review of Psychology. 2007; 58:145-173.

Hensch TK. Critical period plasticity in local cortical circuits. Nature Reviews Neuroscience. 2005; 6:877-888.

Johnson AE, Bruce J, Tarullo AR, Gunnar MR. Growth delay as an index of allostatic load in young children: Predictions to disinhibited social approach and diurnal cortisol activity. Development and Psychopathology. 2011; 23:859-871. [PubMed: 21756437]

Juster RP, McEwen BS, Lupien SJ. Allostatic load biomarkers of chronic stress and impact on health and cognition. Neuroscience \& Biobehavioral Reviews. 2010; 35:2-16. [PubMed: 19822172]

Korte SM, Koolhaas JM, Wingfeld JC, McEwen BS. The Darwinian concept of stress: Benefits of allostasis and costs of allostatic load and the trade-offs in health and disease. Neuroscience \& Biobehavioral Reviews. 2005; 29:3-38. [PubMed: 15652252]

McEwen BS. Protective and damaging effects of stress mediators. New England Journal of Medicine. 1998; 338:171-179. [PubMed: 9428819]

McEwen BS. Central effects of stress hormones in health and disease: Understanding the protective and damaging effects of stress and stress mediators. European Journal of Pharmacology. 2008; 583:174-185. [PubMed: 18282566] 
McEwen BS, Gianaros PJ. Stress- and allostasis-induced brain plasticity. Annual Reviews of Medicine. 2011; 62:431-445.

McEwen BS, Stellar E. Stress and the individual, mechanisms leading to disease. Archives of Internal Medicine. 1993; 153:2093-2101. [PubMed: 8379800]

McEwen BS, Wingfeld JC. The concept of allostasis in biology and biomedicine. Hormones and Behavior. 2003; 43:2-15.

Meaney MJ, Szyf M. Environmental programming of stress responses through DNA methylation: Life at the interface between a dynamic environment and a fixed genome. Dialogues in Clinical Neuroscience. 2005; 7:103-123.

Quevedo K, Johnson AE, Loman ML, LaFavor TL, Gunnar M. The confluence of adverse early experience and puberty on the cortisol awakening response. International Journal of Behavioral Development. 2012; 36:19-28.

Romeo RD, McEwen BS. Stress and the adolescent brain. Annals of the New York Academy of Science. 2006; 1094:202-214.

Shonkoff JP, Boyce WT, McEwen BS. Neuroscience, molecular biology, and the childhood roots of health disparities: Building a new framework for health promotion and disease prevention. Journal of the American Medical Association. 2009; 301:2252-2259. [PubMed: 19491187]

West-Eberhard, MJ. Developmental plasticity and evolution. New York, NY: Oxford University Press; 2003. 


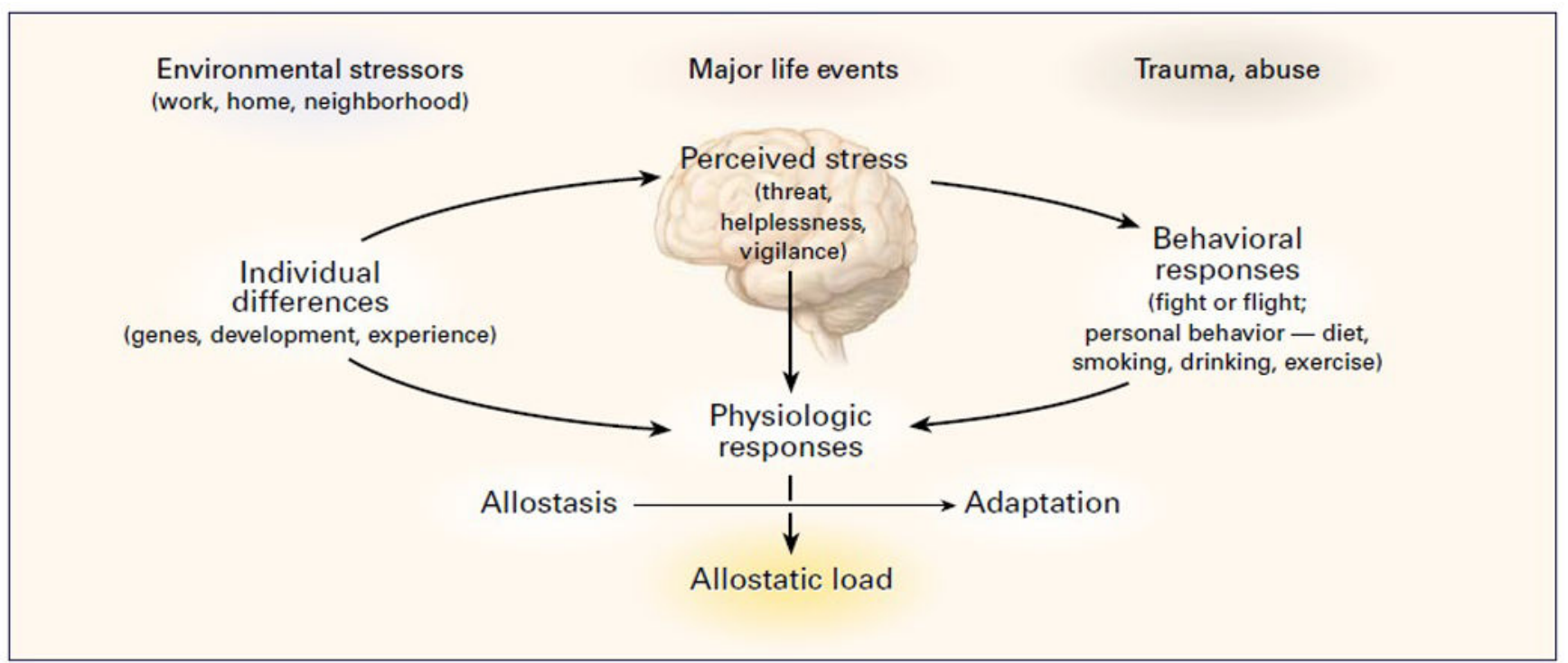

Fig. 1.

Allostatic load model. From "Protective and damaging effects of stress mediators," by B. S. McEwen, 1998, New England Journal of Medicine, 338, p. 172. Copyright $\odot 1998$ by the Massachusetts Medical Society. Reprinted with permission. 


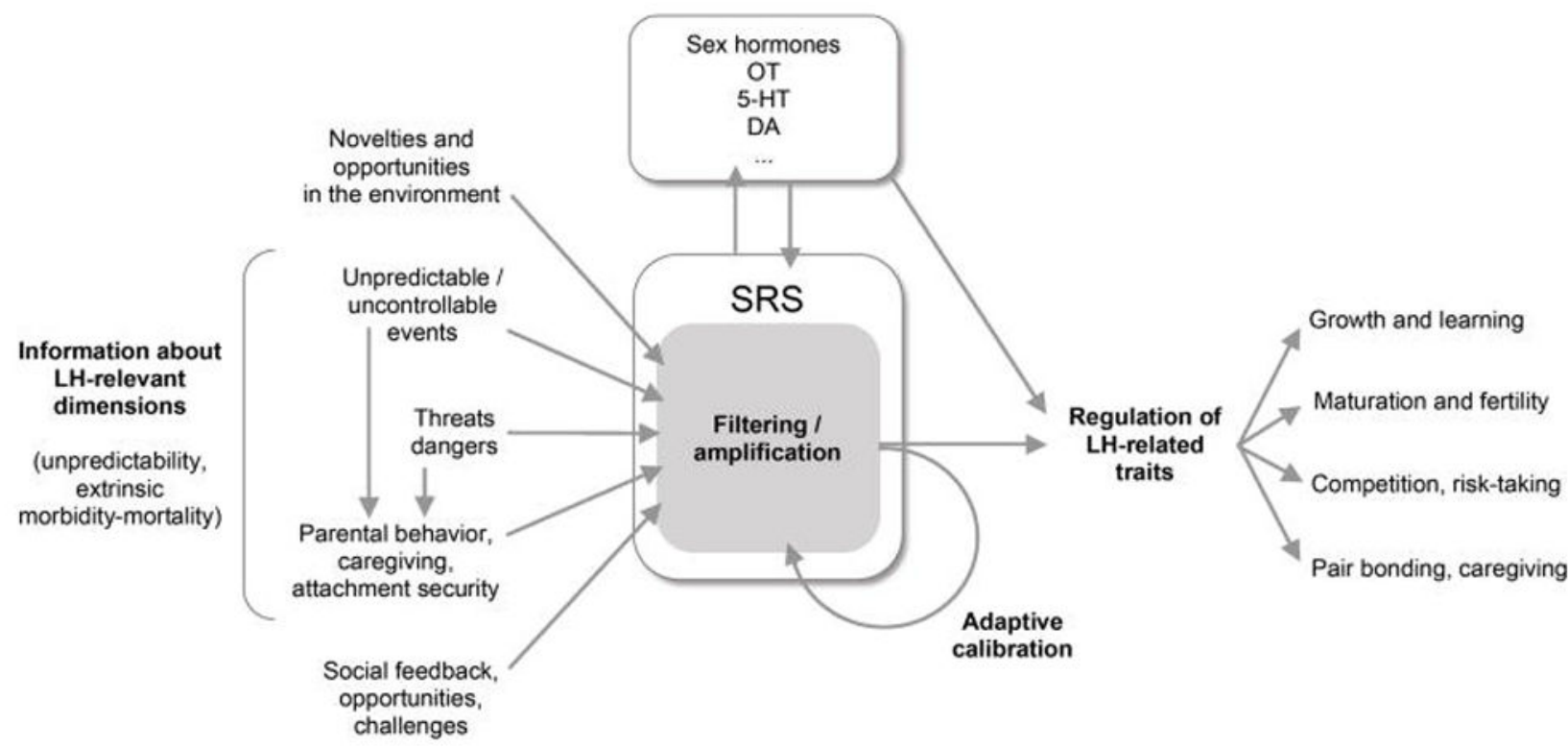

Fig. 2.

Adaptive calibration model. SRS = stress-response system; LH = life history; OT = oxytocin; 5-HT = serotonin; and DA = dopamine. From "The adaptive calibration model of stress responsivity,” by M. Del Giudice, B. J., Ellis, and E. A. Shirtcliff, 2011, Neuroscience \& Biobehavioral Reviews, 35, p. 1564. Copyright ( 92011 by Elsevier Science. Reprinted with permission. 